Revue internationale P.M.E.

Économie et gestion de la petite et moyenne entreprise

\title{
Origines, formes et importance du capital de risque direct: un survol international
}

\section{Jean Marc Suret et Laurence Arnoux}

Volume 8, numéro 3-4, 1995

URI : https://id.erudit.org/iderudit/1008366ar

DOI : https://doi.org/10.7202/1008366ar

Aller au sommaire du numéro

Éditeur(s)

Presses de l’Université du Québec

ISSN

0776-5436 (imprimé)

1918-9699 (numérique)

Découvrir la revue

Citer cet article

Suret, J. \& Arnoux, L. (1995). Origines, formes et importance du capital de risque direct: un survol international. Revue internationale P.M.E., 8(3-4), 233-258. https://doi.org/10.7202/1008366ar
Résumé de l'article

Cet article récapitule les résultats des divers travaux qui, dans le monde anglosaxon, ont étudié le financement direct (ou informel) de la PME. Le financement direct se compose de capitaux fournis par des particuliers, sans garanties, en dehors de tout processus formel d'intermédiation et en l'absence de liens familiaux. Ces particuliers sont connus sous le terme d'anges. Contrairement aux études antérieures, celle-ci aborde le financement direct du point de vue de l'entreprise à la recherche de fonds et tente d'apporter des éléments de réponse à trois questions. 1) Qui sont les anges et quel type d'investissement réalisent-ils ? 2) De quelle manière et sur quels critères ces investisseurs prennent-ils leurs décisions? 3) Quels sont les coûts et conditions de ce mode de financement et quelle est l'offre globale de capital direct? Le survol des études montre que le financement direct est un phénomène important sur le plan économique, bien que son caractère même en rende l'évaluation précise impossible. Les travaux antérieurs permettent de tracer un portrait des investisseurs directs et de caractériser les réseaux non structurés qui les lient. Finalement, l'analyse des modes de fonctionnement des anges permet de dégager certains avantages et problèmes propres au financement direct et de préciser la manière dont les PME devraient aborder ce type de financement.
Ce document est protégé par la loi sur le droit d'auteur. L'utilisation des services d'Érudit (y compris la reproduction) est assujettie à sa politique d'utilisation que vous pouvez consulter en ligne.

https://apropos.erudit.org/fr/usagers/politique-dutilisation/ 


\title{
Origines, formes et importance du capital de risque direct: un survol international ${ }^{1}$
}

\author{
Jean Marc SURET* \\ Laurence ARNOUX** \\ CIRANO, Montréal
}

\section{MOTS CLÉS}

\section{PME - Financement \\ Investissement direct - Capital informel}

\begin{abstract}
RÉSUMÉ
Cet article récapitule les résultats des divers travaux qui, dans le monde anglosaxon, ont étudié le financement direct (ou informel) de la PME. Le financement direct se compose de capitaux fournis par des particuliers, sans garanties, en dehors de tout processus formel d'intermédiation et en l'absence de liens familiaux. Ces particuliers sont connus sous le terme d'anges. Contrairement aux études antérieures, celle-ci aborde le financement direct du point de vue de l'entreprise à la recherche de fonds et tente d'apporter des éléments de réponse à trois questions. 1) Qui sont les anges et quel type d'investissement réalisent-ils? 2) De quelle manière et sur quels critères ces investisseurs prennent-ils leurs décisions ? 3) Quels sont les coûts et conditions de ce mode
\end{abstract}

* Jean Marc Suret détient un doctorat en administration des affaires de l'Université Laval. Il est professeur titulaire au Département de finance de cette université et directeur de recherche au Centre interuniversitaire de recherche en analyse des organisations (CIRANO) de Montréal. Il s'intéresse à la problématique du financement des entreprises, à l'analyse financière et à l'efficience des marchés. Ses articles ont été publiés notamment dans Contemporary Accounting Research, The Financial Review, The Journal of Financial Research, The Geneva Papers on Risk an Insurance, The Canadian Journal of Administrative Sciences, Finance et l'Actualité Économique. Adresse : Centre interuniversitaire de recherche en analyse des organisations, 2020, rue Université, 25e étage, Montréal (Québec) H3A 2 A5.

** Laurence Arnoux, qui détient un MBA en administration des affaires de l'Université Laval, était stagiaire de recherche au CIRANO. Adresse: Centre interuniversitaire de recherche en analyse des organisations, 2020, rue Université, $25^{\mathrm{e}}$ étage, Montréal (Québec) H3A 2A5.

1. Ce document s'inspire de la première section d'une étude menée pour le compte du Conseil de la science et de la technologie du Québec. Voir Suret et Arnoux (1995). 
de financement et quelle est l'offre globale de capital direct? Le survol des études montre que le financement direct est un phénomène important sur le plan économique, bien que son caractère même en rende l'évaluation précise impossible. Les travaux antérieurs permettent de tracer un portrait des investisseurs directs et de caractériser les réseaux non structurés qui les lient. Finalement, l'analyse des modes de fonctionnement des anges permet de dégager certains avantages et problèmes propres au financement direct et de préciser la manière dont les PME devraient aborder ce type de financement.

\section{ABSTRACT}

This research presents a synthesis of the results of past studies on direct (or informal) investment in small business. Direct investment can be characterized as unguaranteed capital provided by individuals without any family relations to the financed businesses and in the absence of any intermediation. The individuals providing the financing are known in the literature as "angels". As contrasted to past publications on the subject, this research adopts the point of view of the company seeking financing and attempts to provide answers to the following typical questions. Who are the angels and what types of investments do they undertake? How do they analyse possible companies for investment, and according to what criteria do they choose the companies financed? What are the conditions and costs of this type of financing, and what is the overall supply of direct capital? The survey indicates that direct investment is a material contribution to small business financing in spite of the impossibility of its precise evaluation. Past research provides a general description of the characteristics of direct investors and the unstructured networks by which they are linked. Finally, the analysis of the methods employed by angels to undertake direct financing activities permits the identification of certain advantages and problems specific to direct investment and some suggestions for small companies wishing to obtain access to this type of financing.

\section{RESUMEN}

Es asi como en este artículo se recopila los resultados de diversos trabajos que, en el medio de habla inglesa, han tomado al financiamiento directo (o informal) de las pequeñas y medianas empresas (PyMEs) como tema principal. El financiamiento directo se caracteriza por ser un capital proveniente de particulares sin ningún vínculo familiar, por no tener ninguna garantía, y por estar fuera de todo proceso formal de intermediación. Estos particulares son conocidos bajo el nombre de "ángeles". A diferencia de estudios anteriores, en este trabajo se adopta el punto de vista de la empresa en busca de fondos, y se intenta responder a las siguientes preguntas: 1) ¿ Quiénes son los llamados ángeles y qué tipo de inversión realizan? 2) ¿ De qué manera y sobre qué criterio éstos toman la decisión de investir en una determinada empresa? 3) ¿Cuáles son los costos y las condiciones de este tipo de financiamiento, y cuál es la oferta global de capital directo?

Se llega a demostrar que el financiamiento directo representa una contribución importante para el tipo de empresa en estudio, a pesar que es imposible una estimación precisa de este tipo de financiamiento. Los estudios 
anteriores permiten revelar las características de los inversionistas directos, y describir la red no estructurada que los unen. Finalmente, el análisis de los métodos utilizados por los "ángeles" permite esclarecer algunas ventajas y problemas específicos del financiamiento directo, además sugiere la forma por la cual las PyMEs deben abordar este tipo de financiamiento.

\section{Introduction}

Que ce soit au Québec, dans le reste du Canada ou dans d'autres pays de l'OCDE, les difficultés de financement par les fonds propres que rencontrent les PME sont régulièrement évoquées et justifient souvent l'intervention des gouvernements. Ainsi, l'étude menée pour le premier ministre de l'Ontario et intitulée Competing in the New Global Economy (1987) rapporte que le manque de fonds propres a constitué une contrainte majeure au développement pour plus de la moitié des entreprises étudiées. Le problème serait particulièrement important dans le domaine des hautes technologies. Le Conseil de la science et de la technologie du Québec écrit (1993, p. 68) que «les PME technologiques et innovatrices rencontrent des difficultés majeures pour le financement de leurs activités de développement [...]». De plus, «la grande majorité des fonds disponibles ont eu tendance, depuis 1988, à délaisser le financement des entreprises technologiques (p. 72)». Selon Knight (1985), qui a étudié le financement de petites entreprises de haute technologie au Canada, ce sont $43 \%$ des sociétés qui font face à de réelles difficultés pour obtenir du financement à un moment ou un autre de leur développement. En France, les difficultés de financement des entreprises de petite taille ont été évoquées par Levratto (1990) et Belletante (1991). Toutefois, d'autres travaux mettent en évidence une offre abondante de capital de risque (Suret, 1994) et de nombreux investisseurs institutionnels déplorent la rareté des propositions d'investissement. L'abondance de l'offre et l'insatisfaction de certaines demandes laissent croire à une situation d'inadéquation, où les investisseurs conventionnels ne seraient pas en mesure de répondre aux demandes des entreprises ${ }^{2}$. On évoque donc la présence de discontinuités des marchés financiers: les gaps. Dans ce contexte, il semble particulièrement intéressant d'étudier un mode de financement assez mal connu qui, suivant divers travaux, impliquerait des montants beaucoup plus élevés que ceux habituellement attribués au capital de risque institutionnel. Il s'agit du financement direct, mis à la disposition des entrepreneurs par ceux

2. Il existe d'autres possibilités d'explication à l'observation simultanée d'une offre abondante et de l'insatisfaction de certaines demandes, comme la réticence au partage du contrôle de la part du dirigeant, la méconnaissance du capital direct par les entrepreneurs, et les coûts élevés que représentent, pour les investisseurs institutionnels, l'analyse et le suivi des dossiers de financement de faible ampleur. 
que l'on nomme désormais les anges ${ }^{3}$. Ce sont des investisseurs privés qui fournissent directement, c'est-à-dire sans l'intermédiaire d'un marché organisé ou d'une institution et généralement sans garanties, des capitaux à des entrepreneurs avec lesquels ils n'ont aucun lien de parenté ${ }^{4}$ et généralement aucune relation avant l'investissement. Le placement se fait le plus souvent sous forme de fonds propres, mais peut inclure des prêts et des cautionnements. Il est généralement réalisé en collaboration avec d'autres investisseurs qui peuvent être directs ou institutionnels.

L'existence et l'importance relative de l'investissement direct changent totalement la manière dont les gouvernements peuvent intervenir pour stimuler la création et le développement des PME, notamment dans le secteur technologique. Comme l'écrivent Riding et al. (1993), " [une fois prise en compte l'offre de capital direct], on ne manque ni de capital, ni d'innovations, ni d'idées; seule manque la connaissance ». Les auteurs font ici référence à la connaissance par les entrepreneurs des modalités et des mécanismes du financement et à celle des projets et de leur valeur, par les investisseurs. Toutefois, notre connaissance des investisseurs directs est, elle aussi, très fragmentaire. Le phénomène est mal connu des entrepreneurs, mais aussi des responsables de l'élaboration des politiques. Nous proposons ici une synthèse de travaux menés sur ce sujet dans le monde au cours des dix dernières années.

C'est aux États-Unis que l'intérêt universitaire pour les investisseurs directs s'est manifesté le plus tôt, et plusieurs études y ont été consacrées, couvrant différentes régions: Nouvelle-Angleterre (Wetzel, 1981), Californie (Tymes et Krasner, 1983), Sunbelt (Gaston et Bell, 1986), région des Grands Lacs (Aram, 1989) et Côte Est (Haar et al., 1988). Une étude plus systématique du phénomène sur l'ensemble du territoire américain a également été menée, avec le concours de l'Office of Economic Research of the US Small Business Administration (Gaston, 1989). Au Canada, Riding et Short (1987) ont étudié la région d'Ottawa-Carleton et d'autres recherches, de plus grande ampleur,

3. Dans ce document, les termes d'investisseur direct et d'ange sont employés indifféremment. Le terme d'investissement direct a été préféré à celui d'investissement informel, qui traduit le vocable américain d'informal investment désormais attaché à ce type d'activité. En effet, l'expression a acquis en français une connotation illégale, à la suite de travaux traitant du secteur informel de l'économie. Par exemple, Verna (1994) définit le secteur informel comme «l'ensemble des activités illégales auxquelles une nette majorité de la population locale reconnaît une certaine légitimité ». Le capital de risque direct n'ayant rien d'illégal, nous avons proposé un terme qui évite toute ambiguité.

4. L'absence de liens familiaux différencie le capital direct du «financement amical» que procurent les proches de l'entrepreneur. Cette distinction est effectuée par la plupart des auteurs, mais n'est pas générale. Haar et al. (1988), par exemple, assimilent les proches de l'entrepreneur à des anges. 
couvrent principalement l'Ontario et les provinces maritimes (Riding et al., 1993). Une étude a porté sur le Québec (Suret et Arnoux, 1995). Quelques recherches ont été menées en Europe, au Royaume-Uni (Mason et al., 1991) et en Suède (Landström, 1993). La situation du capital direct sur le plan international a été décrite par Harrison (1993).

Dans cette étude, nous passons en revue ces diverses recherches, en nous plaçant du point de vue de l'entreprise qui, face à des besoins de financement que pourraient combler les investisseurs directs, doit trouver des réponses à trois questions: 1) Qui sont les anges et quel type d'investissement réalisent-ils? 2) De quelle manière et sur quels critères ces investisseurs prennent-ils leurs décisions ? 3) Quels sont les coûts et conditions de ce mode de financement et quelle est l'offre globale de capital direct? Nous tentons en outre, lorsque cela est possible, de comparer les investisseurs directs aux sociétés de capital de risque (SCR). Toutefois, nous ne décrivons pas ici l'industrie conventionnelle du capital de risque puisqu'il existe, sur ce sujet, de nombreux travaux ${ }^{5}$.

\section{Qu'est-ce que l'investissement direct?}

Les investisseurs directs fournissent généralement des fonds propres à des entreprises de petite taille. Ils offrent donc un produit financier similaire à celui des SCR, dont ils se distinguent par l'absence de toute structure et par le caractère individuel de la démarche.

\subsection{Les investisseurs directs}

Les études menées en Amérique du Nord et en Europe s'accordent globalement sur le profil des investisseurs directs: ce sont presque exclusivement des hommes d'âge mûr, fortunés, ayant un niveau d'études élevé et une expérience des affaires. Le tableau 1 fournit des informations plus précises sur les caractéristiques de ces investisseurs observées dans différents pays et régions.

Les investisseurs directs ont généralement entre 40 et 60 ans. Toutefois, les anges britanniques et suédois sont sensiblement plus âgés que les NordAméricains ${ }^{6}$. La très grande majorité a poursuivi des études universitaires.

5. Voir, par exemple, Geoffron (1991) pour une analyse comparée du processus de structuration de cette industrie en France et aux États-Unis et l'OCDE (1986) pour un survol international.

6. Harrison et Mason (1992a) et Landström (1993) attribuent ce fait aux particularités des systèmes fiscaux européens. Toutefois, comme toutes celles qui sont mentionnées dans ce document, de telles différences peuvent ne pas être significatives. Il est cependant impossible d'en juger sur la base des données fournies dans les divers articles consultés. 
TABLeAU 1

Caractéristiques des investisseurs directs dans différents pays et régions

\begin{tabular}{|c|c|c|c|c|c|c|c|c|}
\hline \multirow[b]{2}{*}{ Caractéristiques } & \multicolumn{2}{|c|}{ Europe } & \multicolumn{4}{|c|}{ États-Unis } & \multicolumn{2}{|c|}{ Canada } \\
\hline & R.U. & Suède & Ensemble & Côte Est & $\begin{array}{l}\text { Grands } \\
\text { Lacs }\end{array}$ & Sunbelt & Ensemble & $\begin{array}{l}\text { Ottawa- } \\
\text { Carleton }\end{array}$ \\
\hline Sexe (\% d'hommes) & 99 & & 95 & 98 & & & 98 & \\
\hline Expérience préalable en gestion (\%) & 57 & 96 & 83 & 87 & $>80$ & & 75 & \\
\hline Avoir net moyen $(000 \$)$ & 546 & $57 \%>909$ & 750 & & $>1500$ & 750 & 1026 & $>754$ \\
\hline $\begin{array}{l}\text { \% de l'avoir net composé } \\
\text { d'investissements directs }\end{array}$ & $\begin{array}{l}34 \%<5 \% \\
21 \%>25 \%\end{array}$ & $\begin{array}{l}17 \%<5 \% \\
34 \%>25 \%\end{array}$ & & $54 \%>25 \%$ & $32 \%<5 \%$ & & $40 \%<10 \%$ & \\
\hline Fréquence d'investissement & $\begin{array}{c}2 \text { tous les } \\
3 \text { ans }\end{array}$ & 1 par an & $\begin{array}{l}2 \text { tous les } \\
3 \text { ans }\end{array}$ & & $\begin{array}{l}2 \text { tous les } \\
3 \text { ans }\end{array}$ & $\begin{array}{l}2 \text { tous les } \\
3 \text { ans }\end{array}$ & 1 par an & 1 par an \\
\hline $\begin{array}{l}\text { Taille de l'échantillon } \\
\text { (nombre d'investisseurs) }\end{array}$ & 86 & 52 & 435 & 121 & 55 & 158 & 279 & 25 \\
\hline
\end{tabular}

(nombre d'investisseurs)

Sources : R.U. : Mason et al. (1991) et Harrison et Mason (1992a) ; Suède: Landström (1993) ; États-Unis : Gaston (1989) ; États-Unis, Côte Est : Haar et al. (1988) ; États-Unis, Grands Lacs : Aram (1989) ; États-Unis, Sunbelt : Gaston et Bell (1986); Canada : Riding et al. (1993) ; Canada, Ottawa-Carleton : Riding et Short (1987 et 1988). Toutes les données exprimées à l'origine en diverses devises ont été converties en \$ US pour faciliter les comparaisons. Elles sont exprimées ici en milliers de dollars. Les résultats des travaux de Wetzel (1983) et Tymes et Krasner (1983), plus anciens, n'ont pas été rapportés.

La notation $60 \%>91$ indique que $60 \%$ des observations dépassent la limite de 91 . 
D'après Riding et al. (1993), près de $70 \%$ sont diplômés du premier cycle et $39 \%$ du second cycle 7 . Les anges ont souvent étudié en administration des affaires ou dans un domaine technique. La plupart d'entre eux possèdent une expérience préalable de gestionnaire: c'est le cas de $75 \%$ des anges au Canada, de plus de $80 \%$ aux États-Unis et de $96 \%$ en Suède (tableau 1). Quand ils n'ont pas de responsabilités en entreprise, les anges sont souvent membres de professions libérales (Haar et al., 1988).

Les investisseurs directs ont un avoir net élevé (tableau 1), mais n'appartiennent pas nécessairement à la catégorie des plus grandes fortunes. Les études américaines, menées au cours des années 1980, situent l'avoir net moyen des anges au-dessus de 750000 dollars $^{8}$ - à l'exception de l'étude de Gaston et Bell (1986), menée dans la Sunbelt - et leur revenu annuel entre 90000 et 250000 dollars. Si, dans l'ensemble, il s'agit de contribuables qui se situent dans les classes à haut revenu sans être toutefois des millionnaires, les anges canadiens semblent faire exception à cette règle. Avec un avoir net de l'ordre de 1,03 million de dollars et un revenu familial moyen de 133000 dollars, les investisseurs canadiens se classent dans des tranches de revenu et de richesse beaucoup plus élevées que celles de leurs homologues américains, sans que l'on puisse expliquer ces différences. Il est possible que le phénomène de l'investissement direct soit plus ancien et plus répandu aux États-Unis, où il impliquerait une population d'investisseurs plus vaste.

La fréquence d'investissement des anges est relativement faible. Ils ne réalisent en moyenne pas plus d'un investissement par an. Toutefois, cette moyenne occulte des différences de comportement entre deux grandes catégories d'investisseurs directs, lesquels peuvent être occasionnels ou quasi professionnels. Les premiers ont réalisé un investissement, parfois deux, parce que l'occasion se présentait. Ils se montrent peu expérimentés dans l'évaluation des projets, investissent de petits montants et adoptent une attitude passive envers leur investissement. Les seconds recherchent continuellement des possibilités de placement et investissent régulièrement. Ils se composent un véritable portefeuille d'investissements directs et sont fréquemment sollicités. Ces anges effectuent une analyse plus formelle des projets qui leur sont soumis et s'impliquent dans la gestion de l'entreprise qu'ils financent. D'après

7. Des pourcentages nettement plus élevés avancés par certaines études peuvent refléter des problèmes d'échantillonnage liés au fait que sont contactés en premier lieu les collègues et ex-étudiants des universitaires qui mènent les recherches. C'est le cas de l'étude de Haar et al. (1988) dont l'échantillon provient à $39 \%$ de listes de diplômés, et qui mentionne une proportion de titulaires de maîtrise supérieure à $56 \%$.

8. Afin de faciliter les comparaisons, tous les montants ont été exprimés en dollars américains, sur la base du taux de change qui prévalait à la fin de la période d'étude. 
Harrison (1993), la plupart des anges britanniques appartiendraient à la première catégorie d'investisseurs, alors que leurs homologues américains et suédois seraient du second type. Postma et Sullivan (1990) montrent cependant que les deux catégories existent aux États-Unis et proposent de considérer une catégorie intermédiaire. Il semble donc clair que les investisseurs directs ne constituent pas un ensemble homogène.

Les anges ont des activités d'investissement diversifiées. Au Canada, 74\% déclarent avoir investi en Bourse et $69 \%$, dans l'immobilier. Par rapport aux autres activités d'investissement, le financement direct semble le plus souvent marginal, et une faible proportion de l'actif lui est consacré. Toutefois, l'investissement direct peut représenter une part non négligeable des revenus. En effet, $33 \%$ des personnes interrogées par Riding et al. (1993) déclarent tirer plus de $50 \%$ de leur revenu de cette forme de placement. Il s'agirait dans ce cas d'anges quasi professionnels, évoqués précédemment.

Pourtant, les anges semblent toujours se distinguer des investisseurs institutionnels ou professionnels par leurs motivations: ils investissent parce qu'ils apprécient jouer un rôle dans la vie des entreprises, éprouvent un certain plaisir à suivre l'évolution de leur société, y voient des occasions de réalisation personnelle. On a aussi avancé que les anges pourraient être motivés par des considérations altruistes: par leurs investissements, ils souhaiteraient aider certains entrepreneurs à démarrer leur entreprise, favoriser le développement de produits utiles à la société ou encore générer des bénéfices économiques pour leur communauté. Haar et al. (1988) estiment que $68 \%$ des investisseurs directs seraient prêts à accepter une diminution de $5 \%$ sur un rendement garanti de $20 \%$ en échange de divers bénéfices non financiers. Sullivan et Miller (1990), qui ont analysé les motivations des anges en leur proposant divers scénarios d'investissement, prétendent que ces derniers ne semblent pas guidés exclusivement par l'objectif de maximisation de la richesse que la théorie financière attribue généralement aux investisseurs. D'ailleurs, l'importance des motivations non financières amène Baty (1991) à qualifier les anges d'investisseurs dilettantes ${ }^{9}$, et Harrison (1993) explique en partie ce comportement par le fait que les anges sont généralement assez riches pour ne pas avoir un besoin absolu des rendements de leurs placements directs. Cependant, ces affirmations qui découlent de l'administration de questionnaires ne semblent pas être appuyées par les faits, puisque les taux de rendement requis par les investisseurs directs et par les SCR diffèrent peu. Ces taux requis apparaissent dans la section consacrée aux coûts de ce type de financement.

9. Baty utilise le terme de recreational investors. 


\subsection{Caractéristiques des investissements directs}

L'investissement direct se caractérise par la faiblesse des montants nominaux. Aux États-Unis, le montant moyen des investissements directs serait inférieur à 50000 dollars. Au Canada, seulement $40 \%$ des placements sont d'un montant inférieur à 75000 dollars, mais ils drainent $85 \%$ des fonds investis et l'investissement moyen serait sensiblement plus élevé au Canada qu'aux États-Unis (118 000 dollars contre 58900 dollars). Il n'en demeure pas moins que les anges investissent des montants nettement inférieurs à ceux des SCR, lesquelles considèrent souvent 250000 dollars comme l'investissement minimal et préfèrent investir des sommes de l'ordre de 750000 dollars (Gaston, 1989, p. 26). Cette idée est clairement illustrée au tableau 2 qui présente la répartition, en fonction du montant nominal, des placements réalisés aux États-Unis par les deux types d'investisseurs.

\section{TABLEAU 2}

Répartition des investissements

des investisseurs directs et des sociétés de capital de risque (SCR) suivant la taille des placements

\begin{tabular}{lccr}
\hline & $\begin{array}{c}\text { Investisseurs } \\
\text { directs, } \\
\text { Côte Est }\end{array}$ & $\begin{array}{c}\text { Investisseurs } \\
\text { directs, } \\
\text { Nouvelle-Angleterre }\end{array}$ & SCR \\
\hline moins de $250000 \$$ & $58 \%$ & $88 \%$ & $5 \%$ \\
de 250000 à $500000 \$$ & $24 \%$ & $7 \%$ & $8 \%$ \\
de 500000 à $1000000 \$$ & $8 \%$ & $5 \%$ & $18 \%$ \\
plus de $1000000 \$$ & $10 \%$ & $59 \%$ \\
\hline
\end{tabular}

Sources: Investisseurs directs, Côte-Est: Haar et al. (1988) - 286 investissements, largement concentrés dans le domaine des services financiers; SCR et Investisseurs directs, Nouvelle-Angleterre - Freear et Wetzel (1990): 177 financements en haute technologie.

Au regard des montants nominaux investis, mais aussi du stade de développement des firmes, il existerait une certaine complémentarité entre les investisseurs directs et les SCR. Les premiers ont tendance à fournir de plus petits montants et sont davantage présents dans les premiers stades de développement des sociétés ; les seconds assureraient les étapes suivantes du financement. D'après Freear et Wetzel (1990), les phases de prédémarrage et de démarrage concentrent $60 \%$ du nombre des investissements directs et $54 \%$ des montants investis sous cette forme dans les firmes de haute technologie situées en Nouvelle-Angleterre. D'après Venture Economics Canada, les proportions seraient respectivement de $28 \%$ et $20 \%$ dans le cas des investissements de SCR en 1990. Carter et Van Auken (1994) rapportent des proportions de $15 \%$ et de $22 \%$, ce qui serait cohérent avec l'hypothèse, exprimée par Venture 
TABLEAU 3

Caractéristiques des investissements directs dans différents pays et régions

\begin{tabular}{|c|c|c|c|c|c|c|c|c|}
\hline \multirow[b]{2}{*}{ Caractéristiques } & \multicolumn{2}{|c|}{ Europe } & \multicolumn{4}{|c|}{ États-Unis } & \multicolumn{2}{|c|}{ Canada } \\
\hline & R.U. & Suède & Ensemble & Côte Est & $\begin{array}{l}\text { Grands } \\
\text { Lacs }\end{array}$ & Sunbelt & Ensemble & $\begin{array}{l}\text { Ottawa- } \\
\text { Carleton }\end{array}$ \\
\hline Taux de rejet & $93 \%$ & $70 \%$ & $88 \%$ & & & $75 \%$ & $97,5 \%$ & $80 \%$ \\
\hline \multicolumn{9}{|l|}{ Stades de développement } \\
\hline Développement (start-up) & $30 \%$ & $27 \%$ & $56 \%$ & $58 \%$ & $55 \%$ & & & \\
\hline Croissance (young) & $51 \%$ & $43 \%$ & $24 \%$ & & $30 \%$ & & & \\
\hline Expansion (established) & $19 \%$ & $30 \%$ & $20 \%$ & & $15 \%$ & & & \\
\hline $\begin{array}{l}\text { Proportion des investisseurs } \\
\text { qui se limitent à un rayon }\end{array}$ & & & & & & & & \\
\hline de $75 \mathrm{~km}$ & $54 \%$ & & $72 \%$ & $58 \%$ & $76 \%$ & $100 \%$ & $53 \%$ & $85 \%$ \\
\hline Montant moyen de & & & & & & & & \\
\hline l'investissement (000 \$ US) & 18,7 & 63 & 58,9 & 130 & & 37,5 & 156 & 82 \\
\hline Taux de rendement attendu & $31 \%$ & $15 \%$ & $22 \%$ & & & $20 \%$ & $32 \%$ & $32 \%$ \\
\hline Horizon d'investissement (années)* & $59 \%<5$ & $41 \%$ & $5-7$ & $75 \%<5$ & & $3-4$ & 6 & 5 \\
\hline
\end{tabular}

Sources: Idem (tableau 1).

* La notation $59 \%<5$ indique que $59 \%$ des répondants ont un horizon inférieur à 5 ans. 
Economics Canada, d'une diminution constante de la proportion des fonds consacrée par les SCR aux opérations de prédémarrage et de démarrage. Cependant, l'intérêt des investisseurs directs pour les opérations de démarrage semble être propre aux investisseurs américains; les anges européens seraient moins enclins à investir dans les premiers stades de développement (tableau 3).

Les investissements directs sont réalisés dans tous les secteurs d'activité. Au Canada, ces placements semblent s'orienter surtout vers les ressources naturelles, la production manufacturière et l'immobilier. Comme le montre la répartition sectorielle des placements des deux types d'investisseurs (tableau 4), les anges seraient moins attirés que les SCR par la haute technologie et la production manufacturière, et davantage par les ressources naturelles. Toutefois, ce tableau montre également que la répartition sectorielle des placements des SCR varie d'une étude à l'autre.

TABLEAU 4

Répartition sectorielle des investissements des investisseurs directs et des sociétés de capital de risque (SCR)

\begin{tabular}{lccc}
\hline & \multicolumn{2}{c}{ SCR } & $\begin{array}{c}\text { Investisseurs } \\
\text { directs }\end{array}$ \\
\hline & $\begin{array}{c}\text { Venture } \\
\text { Economics }\end{array}$ & Jog et al. & Riding et al. \\
\hline Haute technologie & $26 \%$ & $45,42 \%$ & $12 \%$ \\
Biens d'équipement & $26 \%$ & $33,02 \%$ & $15 \%$ \\
$\quad$ et de consommation & $6 \%$ & $11,26 \%$ & $13 \%$ \\
Services & $8 \%$ & & $7 \%$ \\
Construction & $2 \%$ & $4,01 \%$ & $6 \%$ \\
Finance & $3 \%$ & $6,27 \%$ & $17 \%$ \\
Ressources naturelles & $29 \%$ & & $30 \%$ \\
\hline
\end{tabular}

Sources: SCR : Venture Economics et Jog, Lawson et Riding (1991, p. 11); Investisseurs directs: Riding et al. (1993).

* Inclut notamment les transports, le commerce et l'immobilier.

C'est dans le secteur des hautes technologies que l'importance du financement direct a été mise en évidence pour la première fois (Wetzel, 1981). Certains chercheurs le considèrent comme le domaine privilégié des investisseurs directs parce que le manque de fonds y serait plus marqué. Or, au Canada, Riding et al. (1993) estiment que seulement $12 \%$ des placements directs et $26 \%$ des investissements des SCR sont réalisés dans ce secteur. Aux États-Unis, alors que d'après Venture Economics Canada, 39\% des investissements des SCR se feraient dans le domaine des hautes technologies, Haar et al. (1988) rapportent que c'est le cas de $28 \%$ des investissements directs. D'après Gaston (1989), 
seulement $13 \%$ des investisseurs directs américains ont investi dans des entreprises de haute technologie, et $6 \%$ l'ont fait exclusivement dans ce domaine. Au Royaume-Uni, Mason et al. (1991) estiment que ce secteur concentre environ 5\% des investissements directs. L'étude de Landström (1993) menée en Suède tend à montrer que l'investissement direct ne jouerait qu'un rôle limité dans ce domaine. Toutes les études consultées rapportent donc que les investisseurs directs seraient moins attirés que les SCR par le secteur des hautes technologies ${ }^{10}$. Deux raisons peuvent être avancées pour expliquer cette observation. Il est possible que les entreprises de ce secteur, qui demandent souvent des mises de fonds subséquentes considérables, conviennent moins à des individus dont les capacités d'investissement sont forcément moindres que celles d'institutions. Il est, par ailleurs, possible que les investisseurs directs ne disposent pas de l'expertise requise pour analyser des projets de haute technologie. En effet, pour ce type d'investissement, l'expérience antérieure dans le domaine serait déterminante (Wetzel, 1983).

Les investissements directs semblent être des placements de proximité: la majorité des investissements seraient réalisés à moins de $75 \mathrm{~km}$ du domicile ou du bureau du particulier. La (relative) proximité des investissements pourrait être liée au désir d'implication des investisseurs dans les sociétés. En facilitant l'échange d'information, cette proximité permet en outre de réduire l'asymétrie informationnelle, donc les coûts de financement. De plus, les différentes études montrent une large dispersion géographique des anges. Cette répartition contraste avec celle des investissements conventionnels. Les SCR sont fortement concentrées géographiquement, et investissent dans des régions spécifiques; ainsi, pour le Canada, Harrison (1993) note que l'offre est concentrée à Toronto, et dans une moindre mesure à Montréal et à Calgary, et que Toronto attire une grande partie des investissements. Ce phénomène poserait des problèmes d'accès au capital pour les firmes des régions périphériques, d'autant plus qu'elles font également face à un marché du prêt bancaire moins compétitif et parfois plus onéreux (Belletante, 1991). En assurant un financement local et décentralisé, l'investissement direct jouerait donc un rôle important dans le développement régional.

\section{Comment procèdent les investisseurs directs?}

Aborder le financement direct demande, de la part de l'entreprise, une connaissance des comportements et du processus de décision de cette catégorie

10. Toutefois les anges manifesteraient un vif intérêt pour investir dans le secteur des hautes technologies, peut-être pour des raisons de prestige. D'après Gaston (1989), près d'un quart serait très intéressé à investir dans ces activités. Riding et Short (1987) rapportent que ce secteur serait le premier considéré $(37,5 \%)$ pour des investissements ultérieurs. 
particulière d'investisseurs. Nous nous intéresserons donc successivement à l'existence de réseaux d'investisseurs, aux critères qu'ils privilégient pour accepter ou refuser un financement et, finalement, aux méthodes qu'ils emploient pour contrôler le risque.

\subsection{Les anges et leur réseau}

Les anges n'opèrent pas de manière isolée, même lorsque ce ne sont que des investisseurs occasionnels. Ils sont reliés par des réseaux informels, qui jouent un rôle dans la transmission de l'information relative aux occasions de placement, dans l'analyse de ces possibilités d'investissement et dans l'élaboration de montages financiers permettant aux anges de réduire le risque de leur portefeuille en diversifiant les placements.

Les études sont unanimes pour affirmer que les réseaux qui lient les anges constituent la principale source d'information concernant les occasions d'investissement. Aux États-Unis, amis et associés d'affaires seraient à l'origine de plus de $41 \%$ des occasions d'affaires et de $77 \%$ des investissements réalisés (Freear et Wetzel, 1989). Au Canada, les associés d'affaires créent $60 \%$ des occasions d'investissement; de plus, $70 \%$ des investisseurs interrogés par Riding et al. (1993) affirment recommander régulièrement les entrepreneurs à d'autres investisseurs. Le niveau élevé d'efficacité de ces réseaux semble être une caractéristique nord-américaine; au Royaume-Uni, l'information véhiculée serait de moins bonne qualité (Harrison et Mason, 1992a). Partout, les réseaux d'investisseurs ne constituent pas la seule source d'information. La recherche personnelle active fournit également de nombreuses occasions d'affaires. Celles-ci se concrétisent cependant moins souvent que celles qui découlent des autres sources.

Les interactions entre les investisseurs seraient également importantes dans la prise de décision: les recommandations de certains anges semblent influencer largement les choix d'autres investisseurs. Les relations seraient plus étroites encore au niveau du financement. Les anges investissent le plus souvent avec d'autres particuliers, dans le cadre de syndicats qui sont rarement structurés de manière formelle. Selon Gaston (1989), 11 anges américains sur 12 investissent avec d'autres particuliers. Au Canada, $75 \%$ des investissements directs concernent plus d'un particulier (Riding et al., 1993). Les investissements syndiqués concerneraient des montants plus élevés. Les anges britanniques, qui agissent de manière plus indépendante - seulement $44 \%$ investissent avec d'autres particuliers (Harrison et Mason, 1992a) -, semblent constituer une exception.

Les implications pour les PME à la recherche de fonds sont claires: il leur faut repérer puis contacter ces réseaux. Comme c'est le cas pour les 
chercheurs qui ont étudié le phénomène, l'identification du ou des premiers investisseurs directs est l'étape la plus difficile. Ensuite, il est relativement aisé de connaître l'ensemble du réseau. Certains membres des communautés d'affaires sont généralement en mesure d'identifier un ou des investisseurs directs : responsables de SCR, commissaires industriels, responsables de sociétés de développement régional, gestionnaires de patrimoine. Ils sont donc des sources d'information privilégiées pour les entreprises à la recherche de fonds. La difficulté de contacter les investisseurs informels n'est cependant qu'une des dimensions de «l'écart de connaissance» qui sépare les promoteurs des investisseurs. L'autre dimension, souvent mentionnée par les investisseurs directs que nous avons pu rencontrer, tient au fait que les dirigeants d'entreprises ne connaissent pas les modalités de fonctionnement du capital de risque. La détermination d'un taux de rendement normal compte tenu du niveau de risque, l'établissement de la participation de l'investisseur aux décisions et les modalités de sortie de l'investissement sont autant de pierres d'achoppement du processus de négociation.

\subsection{Le processus de décision}

Riding et al. (1993) ont étudié en détail le processus de décision des investisseurs directs. Ils le décomposent en trois étapes: prise de connaissance des occasions d'investir, évaluation du projet et rencontre avec le promoteur. Les critères avancés par les anges comme les plus importants pour motiver leur décision sont, à chacune des étapes du processus, le potentiel du produit, le rendement prévu du projet, l'expérience et la formation des promoteurs ainsi que les perspectives perçues du secteur d'activité. Il ne semble pas exister de différences très nettes entre les pondérations accordées à ces différents critères selon le stade du processus. Parmi les autres critères jugés importants par une large proportion d'investisseurs, on peut noter le réalisme du projet et, à la fin du processus, l'existence d'un plan d'affaires. Il semble toutefois que les anges accordent moins d'importance aux documents formels concernant le projet que les SCR: seulement $3 \%$ d'entre eux citent le plan d'affaires comme critère de décision le plus important (Haar et al., 1988). Les anges font, dans une large mesure, confiance à leur intuition et ont tendance à investir dans ce qu'ils connaissent au regard du produit, de la technologie et du marché.

La personnalité des entrepreneurs et leurs qualités de gestionnaires apparaissent comme des critères déterminants. Ainsi, $80 \%$ des anges interrogés par Riding et al. (1993) affirment avoir rejeté des propositions d'investissement à cause d'un manque de confiance dans les capacités managériales des entrepreneurs, ce qui permet aux auteurs d'affirmer «les investisseurs directs investissent autant dans l'entrepreneur que dans l'entreprise» (p. 27). Le 
manque de compétences attribué aux gestionnaires est perçu comme le principal facteur de risque lié aux projets et comme le principal obstacle à la multiplication des placements.

Selon les pays, à l'issue du processus d'analyse, de 7 à $30 \%$ des projets soumis aux investisseurs directs sont financés. Les anges canadiens apparaissent particulièrement sélectifs, avec un taux d'acceptation inférieur à celui des anges américains et européens (tableau 3). Harrison et Mason (1992a) font remarquer qu'avec un taux de rejet de $93 \%$, les anges britanniques restent moins sélectifs que les SCR. Dans la mesure où ils rejettent plus de projets que les anges américains et ont sensiblement la même fréquence d'investissement, les auteurs en déduisent que les investisseurs britanniques reçoivent davantage de propositions, mais que celles-ci seraient de moins bonne qualité. Les auteurs concluent également que les anges sont moins expérimentés et moins professionnels au Royaume-Uni lorsqu'il s'agit de repérer les propositions dignes d'intérêt.

\subsection{Le contrôle du risque}

Les investissements directs présentent un niveau de risque élevé, qui combine les aléas de la performance de la firme et la possibilité d'expropriation par l'entrepreneur ou d'autres investisseurs et la faible diversification inhérente à l'absence de processus d'intermédiation. Un degré d'aversion modéré pour le risque paraît être l'une des caractéristiques des investisseurs directs ; toutefois, ceux-ci ne consacrent généralement qu'une faible proportion de leur portefeuille à ces activités. De plus, ils mettent en œuvre plusieurs types de stratégie leur permettant de contrôler le risque.

Une des premières méthodes de réduction du risque concerne la forme du placement. Ainsi, les investisseurs canadiens exigent un contrat d'actionnaire formel $(90 \%)$, négocient des clauses restrictives $(62 \%)$ et prennent dans les entreprises des positions mixtes de créanciers et d'actionnaires (72\%) (Riding et al., 1993). Aux États-Unis, Wetzel (1983) note que $47 \%$ des anges exigent généralement une clause concernant le rachat des actions. Le manque de liquidité de ce type de placement est en effet une source de risque. Landström (1993) fait remarquer que la revente aux entrepreneurs est fréquente aux États-Unis, alors qu'il n'existerait pas de mode de sortie adéquat en Suède, ce qui oblige l'investisseur à détenir son placement plus longtemps. Les investisseurs informels semblent utiliser le réseau qui les lie pour réduire le risque rattaché à la faible diversification des portefeuilles, puisque de nombreux placements sont effectués conjointement par deux ou plusieurs investisseurs. 


\section{Les coûts et l'offre de financement direct}

\subsection{Les coûts}

Comme pour tout mode de financement, le coût du capital direct a trois composantes: la première est la rémunération du capital, qui dépend du taux de rendement exigé par l'investisseur. La seconde est un niveau de contraintes plus ou moins élevé induit par les exigences de l'investisseur. Le troisième est un coût de négociation, proportionnel au temps et à la complexité des démarches. Finalement, il faut prendre en compte un bénéfice intangible de cette forme de financement, qui découle de l'implication de gestionnaires souvent expérimentés dans des petites entreprises en expansion.

\subsubsection{Le taux de rendement exigé}

L'objectif premier des anges est d'ordre financier. Ils investissent d'abord parce qu'ils attendent un taux de rendement élevé. Le tableau 3 montre que les taux requis varient suivant les pays. Les investisseurs canadiens prévoient un taux de rendement annualisé de $32 \%$ et leurs homologues britanniques un taux de $31 \%$. Ces taux semble largement supérieurs à ceux de $22 \%$ et de $15 \%$ exigés respectivement par les anges américains et suédois. Il est possible que ces différences soient liées au climat économique et, notamment, aux divergences de taux d'intérêt prévalant dans chacun des pays au moment des études.

Dans le cas de la Suède, le taux de rendement faible en regard des normes habituelles de l'industrie du capital de risque peut être rapproché du fait que seulement $27 \%$ des placements se font au niveau du démarrage (contre $56 \%$ aux États-Unis). Finalement, il est probable que les configurations des systèmes nationaux de financement influencent les taux de rendement requis, donc le coût de financement. L'existence d'un vaste bassin de capital de risque disponible et largement subventionné, tel qu'il apparait au Canada ${ }^{11}$, pourrait conduire à l'abaissement des taux requis soit par l'effet de la concurrence entre les investisseurs, soit parce que l'offre abondante de capital institutionnel facilite la réalisation des investissements directs. À notre connaissance, ces effets n'ont pas été étudiés jusqu'à maintenant.

Bien qu'elle soit parfois pratiquée, la comparaison des taux requis par les anges et les SCR est délicate. En effet, on a montré que les deux types d'investisseurs placent des montants fort différents dans des entreprises dont

11. Les travaux les plus récents (McDonald et Associés, 1994) évaluent les liquidités disponibles dans l'industrie du capital de risque canadienne à 1,885 milliard de dollars canadiens. Ces excédents sont largement imputables aux fonds d'investissements syndicaux, qui bénéficient de privilèges fiscaux considérables. 
les niveaux de risque ne sont pas les mêmes. Les coûts d'analyse et de suivi des dossiers ne sont pas identiques dans l'un ou l'autre cas. On peut donc difficilement comparer globalement les taux exigés, comme le font Freear, Sohl et Wetzel (1990). Ceux-ci observent que le taux de rendement médian exigé par les anges est de $32,5 \%$, alors qu'il serait de $40 \%$ pour les SCR. Le coût du financement direct serait donc nettement moins élevé que celui du capital de risque conventionnel, ce que ces auteurs expliquent par l'importance des considérations non financières pour les anges. Toutefois, l'étude de Jog, Lawson et Riding (1991) rapporte, pour 29 SCR canadiennes, des taux de rendement attendus qui varient entre $27,5 \%$ et $39 \%$ pour les financements en phase de croissance et de $36,4 \%$ pour les phases de démarrage. Suivant ces résultats, il n'existerait donc plus de différence systématique entre les deux catégories d'investisseurs en capital de risque. Les investisseurs informels seraient même plus exigeants que les SCR lors des phases de démarrage. Le tableau 5 récapitule ces divers résultats. Il tient compte des types de financement, mais il doit être interprété avec prudence, puisque la définition des stades de développement ne fait pas l'unanimité parmi les répondants à ce type d'étude, comme le montrent clairement Ruhnka et Young (1987). Il semble donc qu'il soit impossible, dans l'état actuel des travaux, de confirmer empiriquement l'affirmation selon laquelle les investisseurs informels demandent des taux de rendement inférieurs à ceux requis par les SCR pour un niveau de risque identique.

\section{TABLEAU 5 \\ Risque de défaut et taux de rendement requis \\ par les investisseurs directs et par les sociétés de capital de risque suivant diverses études}

\begin{tabular}{lcccccc}
\hline & $\begin{array}{c}\text { Investisseurs directs } \\
\text { (Wetzel, 1981) }\end{array}$ & $\begin{array}{c}\text { Investisseurs } \\
\text { directs } \\
\text { (Freear et } \\
\text { Wetzel, 1989) }\end{array}$ & $\begin{array}{c}\text { SCR } \\
\text { (Ruhnka et Young, } \\
\text { 1987) }\end{array}$ & $\begin{array}{c}\text { SCR } \\
\text { (Jog } \text { et al. } \\
\text { 1991) }\end{array}$ \\
\cline { 2 - 7 } & $\begin{array}{c}\text { Risque } \\
\text { de défaut* }\end{array}$ & $\begin{array}{c}\text { Taux de } \\
\text { rendement }\end{array}$ & $\begin{array}{c}\text { Taux de } \\
\text { rendement }\end{array}$ & $\begin{array}{c}\text { Risque } \\
\text { de défaut** }\end{array}$ & $\begin{array}{c}\text { Taux de } \\
\text { rendement }\end{array}$ & $\begin{array}{c}\text { Taux de } \\
\text { rendement }\end{array}$ \\
\hline Prédémarrage & $70 \%$ & $50 \%$ & $85 \%$ & $66,2 \%$ & $73 \%$ & \\
Démarrage & $60 \%$ & $50 \%$ & $51 \%$ & $53 \%$ & $54,8 \%$ & $36,4 \%$ \\
Croissance & $50 \%$ & $37,5 \%$ & $41 \%$ & $33,7 \%$ & $42,2 \%$ & $27,5 \%$ \\
Expansion & $40 \%$ & $30 \%$ & $30 \%$ & $20,9 \%$ & $35 \%$ & $36,4 \%$ \\
Sortie & $20 \%$ & $22,5 \%$ & $27 \%$ & $20,9 \%$ & $35 \%$ & $39 \%$ \\
\hline
\end{tabular}

* Le risque de défaut correspond au nombre de firmes anticipé par l'investisseur pour lesquelles la perte aurait été supérieure à $50 \%$ du montant investi, sur un portefeuille de 10 projets d'investissement qui répondraient à ses critères.

Les investisseurs directs sont relativement satisfaits des performances de leurs placements, particulièrement aux États-Unis. Gaston (1989) rapporte 
que $28 \%$ des investisseurs américains seulement ont obtenu des rendements inférieurs à leurs attentes. Toutefois, l'expérience des Européens est plus décevante: au Royaume-Uni, $41 \%$ des anges seraient peu satisfaits de la performance de leurs investissements directs (Harrison et Mason, 1992a). Même avec des taux de rendement attendus moins élevés, $25 \%$ seulement des anges suédois estiment avoir obtenu des rendements supérieurs à leurs prévisions, ce qui peut s'expliquer par un climat économique défavorable (Landström, 1993).

\subsubsection{Le coût des contraintes}

On connaît peu de choses sur la façon dont les anges structurent formellement leur entente avec les entreprises. Il semble toutefois que ces investisseurs tentent, dans de nombreux cas, de limiter leur niveau de risque par des moyens autres que les conventions formelles et les contrôles imposés à l'entreprise et, dans ce cas, exercent des contraintes moins strictes sur l'entreprise ${ }^{12}$. Contrairement aux SCR, les investisseurs directs ne cherchent pas nécessairement à concentrer des droits de vote: Riding et Short (1987) rapportent que dans 5\% seulement des cas, l'investisseur a le contrôle de la société et que, dans $53 \%$ des cas, il détient moins de $10 \%$ des droits de vote. De plus, les anges sont généralement considérés comme des investisseurs patients qui fournissent un financement à long terme (Wetzel, 1983; Harrison, 1993; Riding et al., 1993). Cependant, les réponses aux questions précises concernant l'horizon de placement ne permettent pas de les distinguer nettement des SCR. Les diverses études rapportent que les investisseurs directs ont l'intention de détenir leur placement durant une période généralement comprise entre 3 et 7 années $(6,35$ au Canada). Lorsqu'elles investissent dans des phases de prédémarrage ou de démarrage, les SCR rapportent des horizons de 7,2 ans et de 8 ans au Canada (Jog et al., 1991). D'ailleurs, Freear, Sohl et Wetzel (1990) soulignent que l'horizon d'investissement serait similaire à celui des SCR. De manière générale, il semble tout de même que les contraintes supplémentaires imposées à l'entreprise soient relativement moins lourdes lorsque ce sont des investisseurs directs qui interviennent.

\subsubsection{Les coûts de négociation}

Les processus d'analyse et de négociation sont généralement plus rapides lorsqu'ils font intervenir des anges plutôt que des SCR. Ainsi, Freear, Sohl et

12. Pour une revue des analyses comparatives de l'implication des anges et des SCR dans la gestion des entreprises, voir Ehrlich et al. (1994). Ceux-ci concluent (p. 80) que les SCR sont plus susceptibles de contrôler fermement l'entreprise que les investisseurs directs. 
Wetzel (1990) indiquent que la durée médiane qui s'écoule entre la décision de recherche de financement et l'obtention des fonds par les firmes est de 4 mois lorsqu'ils sont accordés par des investisseurs directs et de 6 mois lorsqu'ils proviennent de SCR. Curieusement, le temps qui s'écoule avant la première rencontre avec l'investisseur est inférieur lorsqu'il s'agit d'un ange, alors que ces personnes sont souvent considérées comme plus difficiles à localiser du fait d'un manque d'information. Après la première rencontre, il ne s'écoulerait que 2,5 mois avant l'obtention des fonds quand un investisseur direct est impliqué, et 4,5 mois quand il s'agit d'une SCR. Ces chiffres accréditent l'idée selon laquelle les anges prennent des décisions rapides. Pourtant, la réduction des délais ne semble pas être une motivation des firmes pour solliciter des anges; les deux principales raisons invoquées étant un coût moindre et un besoin de fonds de faible montant (Freear et al. 1990).

\subsection{Les autres avantages de l'investissement direct}

Les anges n'imposent généralement pas de nombreuses contraintes aux gestionnaires dans le but de surveiller leur investissement. Une importante minorité (environ 30\%) a d'ailleurs une attitude passive à l'égard de leurs placements, que ce soit au Canada (Riding et Short, 1987), aux États-Unis (Freear et Wetzel, 1990) ou au Royaume-Uni (Mason et al., 1991). Par contre, les investisseurs suédois ne seraient que $7 \%$ à être inactifs. Lorsque les anges surveillent activement leurs investissements, c'est en s'impliquant dans la gestion de la société. D'après Freear et Wetzel (1990), $25 \%$ fournissent des services de consultant, $23 \%$ siègent sur le conseil d'administration, et $22 \%$ travaillent à temps plein ou partiel pour la société. Ces investisseurs sont donc, pour l'entreprise, d'une contribution qui dépasse l'apport de fonds. Harrison et Mason (1992b), qui ont étudié en détail les modes d'implication des deux types d'investisseurs, estiment que les anges ont un champ d'intervention plus large que les SCR. Toutefois, ils manifestent une prédilection pour le développement des produits et des marchés (évaluation du marché, développement du produit, plan marketing, relations avec les clients), alors que les SCR interviendraient davantage au niveau du contrôle des performances d'exploitation et de la gestion financière. Pour Harrison et Mason (1992b), les anges ne seraient pas plus utiles à la firme que les SCR si l'on évalue la qualité de leur contribution d'ensemble. C'est également la constatation de Freear, Sohl et Wetzel (1990) qui rapportent que $74 \%$ des firmes jugent très productives ou assez productives leurs relations de travail avec les anges, alors que la proportion atteint $78 \%$ pour les représentants des SCR. Les anges ont pourtant une attitude différente: ils gèrent et contrôlent l'entreprise plutôt que leur investissement (Harrison et Mason, 1992b) et sont perçus comme comprenant mieux les comportements et les motivations de l'entrepreneur (Freear et al., 1990). 


\subsection{L'offre de capital direct}

Dans les pays où le phénomène a fait l'objet de quelques études (États-Unis, Canada et Royaume-Uni), l'investissement direct apparaît désormais comme la source de capital de risque à laquelle on a le plus recours, devançant les sociétés institutionnelles. C'est toutefois la seule certitude qui s'impose concernant l'étendue de l'offre de capital de risque direct. En effet, on ne dispose pas de données tangibles sur le phénomène. Il s'agit donc de faire des estimations. Les recherches dans ce domaine sont d'autant plus délicates à mener que les anges se montrent discrets.

Plusieurs estimations ont été réalisées aux États-Unis; quelques hypothèses ont également été formulées au Canada, alors qu'au Royaume-Uni on dispose seulement d'indices sur l'ampleur de l'offre ${ }^{13}$. Selon les auteurs, l'offre de capital direct serait aux États-Unis de 25 à 300 milliards de dollars, et atteindrait peut-être 1 milliard de dollars au Canada (tableau 6). Les disparités s'expliquent par les méthodes utilisées et les hypothèses retenues. Trois approches ont été employées ${ }^{14}$. La première utilise des données sur les finances de ménages et évalue la part consacrée aux investissements dans des sociétés privées. Il est toutefois troublant de constater qu'à partir des mêmes données (Survey of Consumer Finance, 1983), Avery et Elliehausen (1986) et Ou (1987) aboutissent à des estimations très différentes. La seconde méthode vise à déterminer, à partir d'une enquête auprès d'anges identifiés, quelles sont leurs caractéristiques, puis d'estimer le nombre de personnes qui possèdent ce profil. Il ne s'agit alors que d'investisseurs potentiels. L'offre globale est évaluée en considérant qu'une partie de cette population consacre une certaine fraction de son actif à des activités d'investissement direct. La troisième approche consiste à mener une enquête auprès d'entreprises pour déterminer la part du financement fournie par des particuliers.

Le marché potentiel du capital direct serait plus important encore. Il semblerait que la plupart des anges ne trouvent pas suffisamment d'occasions d'investissement répondant à leurs critères. Ils disposeraient de fonds pour d'autres investissements. Parmi les anges interrogés par Riding et al. (1993), $61 \%$ estiment que les projets qui les intéresseraient vraiment sont rares. D'après Gaston (1989), 54\% des anges américains désireraient investir plus que ce que leur permettent les occasions existantes. De plus, le nombre d'anges

13. Pour une revue des estimations de l'offre de capital direct, voir Freear et Wetzel (1991) pour les États-Unis et Harrison et Mason (1990) pour le Royaume-Uni.

14. Il est à noter que la méthode utilisée par Riding et Short (1988), qui apparaît comme la plus rigoureuse et qui s'inspire des techniques employées en biologie, ne se classe dans aucune de ces trois catégories. Elle n'a toutefois été appliquée qu'à la région d'Ottawa-Carleton. 
TABLEAU 6

Estimation de l'offre de capital de risque direct, suivant diverses études

\section{Étude}

Méthode d'estimation

Offre de capital direct

\section{États-Unis}

Wetzel (1987) - À partir de l'étude de l'AEG (1986)* qui couvre $28,6 \%$ de la population

- À partir des estimations d'Avery et Elliehausen (1986)**: hyp. : 1/3 des fonds investis est du capital direct

- Nombre de millionnaires $>1 \mathrm{M}$

\section{hyp. : - actif net moyen: 1-2 M} - 1/4 intéressés par le capital direct - $10 \%$ de l'actif investi
Survey of Consumer Finance (1983): $2 \mathrm{M}$ de ménages ont investi $300 \mathrm{MM} \$$ dans des sociétés privées

$\mathrm{Ou}(1987)$

Gaston et Bell (1988) Inférence fondée sur une enquête menée auprès d'un échantillon de 435 anges relevés à partir d'entreprises du Dun's Market Identifier File

\section{5-62 MM \$, par 345000 anges}

\section{MM \$}

\section{5-50 MM \$}

( 2 fois plus de montants investis et 5 fois plus de firmes financées que les SCR)

\section{$300 \mathrm{MM}$ \$}

(150 fois plus de montants investis que les SCR)

$32,7 \mathrm{M} \mathrm{\$} \mathrm{/} \mathrm{an} \mathrm{par} 720000$ anges (10 fois plus de montants investis que les SCR, 40 fois plus d'entreprises financées)

\section{Canada}

Riding et al. (1993) À partir de leur enquête (taux de réponse: 42,3\%): investissements annuels: 34,9M \$ hyp. : tous les anges ont été identifiés au Canada

Min. : $82,5 \mathrm{M} \mathrm{\$ /an}$ peut-être $500 \mathrm{M}-1 \mathrm{MM} \$$ de fonds investis.

\section{Royaume-Uni}

Cross (1981)

Hakim (1989)

Échantillon de firmes manufacturières en démarrage en Écosse Enquête auprès de 2000 entreprises de moins de 50 salariés (M.A.S. Business Line Survey)

Cary (1989)
$6,3 \%$ du financement

Capital-actions à $2 \%$ des firmes

Source de fonds la plus importante

M : millions ; MM : milliards.

* L'étude de l'Applied Economics Group (1986), menée dans la Sunbelt, évalue le nombre d'anges à 99000 et les sommes investies en capital direct à 5,1 milliards de dollars.

** Avery et Elliehausen (1986), à partir des données de la Survey of Consumer Finance (1983), estiment que 1,3 million de ménages ont investi 151 milliards de dollars dans des entreprises privées où ils ne participent pas à la gestion. 
actifs serait largement dépassé par celui de particuliers ayant un profil similaire, mais qui n'investissent pas. Wetzel et Freear (1994) laissent entendre qu'ils peuvent être deux fois plus nombreux. Au Canada, l'étude de Riding et Short (1988) montre que $5 \%$ seulement des individus possédant les caractéristiques des anges investissent. Riding et al. (1993) font remarquer que 100000 familles ont un niveau de revenu similaire à celui des anges au Canada, représentant une offre potentielle de capital considérable. Ces individus ne seraient pas actifs à cause de leur perception du risque. Riding et al. (1993) estiment que les entrepreneurs pourraient les inciter à investir à condition de les rassurer sur le niveau de risque de leurs projets et de les convaincre de leurs capacités.

\section{Conclusion}

Suivant les résultats des diverses études qui leur ont été consacrées, les investisseurs directs représentent une source importante de financement pour l'entreprise en démarrage et en croissance. La valeur de l'investissement direct est d'abord numérique. Aux États-Unis, où les estimations de l'offre de capital direct ont été les plus nombreuses, elle représenterait de 2 à 10 fois les montants placés par les sociétés de capital de risque et toucherait 5 à 40 fois plus d'entreprises. L'importance de l'investissement direct est également liée à sa complémentarité par rapport aux sources de financement conventionnelles: il s'agit généralement de faibles montants de capital non garanti qui s'accompagnent d'un apport de compétences externes. En effet, les anges s'impliquent souvent dans l'activité de l'entreprise, dans des fonctions liées aux produits et aux marchés.

L'existence des investisseurs directs a des effets considérables sur les politiques économiques. Actuellement, leur poids n'est pas reconnu et l'offre de capital informel n'est pas prise en compte lors des analyses de l'offre et de la demande de capital de risque. Or, particulièrement au Canada, de nombreux énoncés de politiques et programmes reposent sur l'idée que les difficultés de financement des PME sont imputables à l'insuffisance de l'offre de capital. Ces analyses ont conduit, notamment au Québec, à subventionner massivement la création de SCR. La connaissance du financement direct, sa stimulation éventuelle au moyen de la fiscalité et la structuration de mécanismes d'échanges d'information sont vraisemblablement des actions moins coûteuses et plus efficaces que celles qui consistent à créer de nouvelles institutions. En effet, contrairement aux SCR, les investisseurs directs offrent une forme de financement relativement souple, capable de pourvoir aux besoins d'entreprises lors des phases de prédémarrage et de démarrage, et pour des montants limités. 
Malgré l'existence de plusieurs études menées dans le domaine de l'investissement direct, de nombreuses questions subsistent. En effet, l'estimation de l'offre de capital direct reste délicate, et les chiffres avancés peu justifiés. De plus, des questions plus précises, concernant notamment le stade de développement des projets financés, l'importance du capital direct pour le secteur des hautes technologies ou encore le comportement des anges par rapport aux considérations fiscales n'ont pas été éclaircies. La question principale découle cependant de la constatation suivante: l'offre actuelle et potentielle de capital direct est abondante, mais le taux de rejet des projets est extrêmement élevé, ce qui indique que des entreprises à la recherche de capital n'en obtiennent pas. Cette situation provient probablement de décalages entre les attentes des investisseurs directs et celles des entrepreneurs, c'est-à-dire d'un «écart de connaissance» (Riding et al., 1993). Son origine et les moyens de le réduire devraient constituer des sujets de recherche intéressants dans les années à venir. Il s'agit vraisemblablement de l'une des clés de la problématique du financement de l'entreprise en croissance.

\section{Bibliographie}

ARAM, J.D. (1989), "Attitudes and behaviors of informal investors toward EarlyStage investments, technology-based ventures and coinvestors», Journal of Business Venturing, vol. 4, p. 333-347.

AVERY, R.B. et G.E. ElLIEHAUSEN (1986), «Financial characteristics of high income families», Federal Reserve Bulletin, vol. 72, n 3, p. 163-177.

BATY, G.B. (1991), Entrepreneurship for the 1990s, Englewood Cliffs, N.J., PrenticeHall.

Belletante, B. (1991), «Pour une approche des spécificités financières de la PME au travers du concept de territoire financier», Revue Internationale PME, vol. $4, n^{0} 1$, p. 49-79.

CARTER, R.B. et H.E. VAN AUKen (1994), «Venture capital firms' preferences for projects in particular stages of development», Journal of Small Business Development, janvier, p. 60-73.

CARY, L. (1989), The Venture Capital Report Guide to Venture Capital in the U.K., $4^{\mathrm{e}}$ édition, Londres, Pitman.

CONSEIL DE LA SCIENCE ET DE LA TECHNOLOGIE (1993), Urgence technologique: Pour un Québec audacieux, compétitif et prospère, Québec.

CONSEIL DU PREMIER MINISTRE DE L'ONTARIO (1987), Competing in the New Global Economy, Toronto, Gouvernement de l'Ontario.

Cross, M. (1981), New Firm Formation and Regional Development, Farnborough, Gower. 
Ehrlich, S.B., A.F. De Noble, T. Moore et R.R. Weaver (1994), «After the cash arrives: a comparative study of venture capital and private investor involvement in entrepreneurial firms », Journal of Business Venturing, vol. 9, p. 67-82.

FREeAR, J. et W.E. WeTzEL JR (1989), «Equity capital for entrepreneurs», dans K. Vesper (éd.), Frontiers of Entrepreneurship Research, Wellesley, Mass., Babson College.

FreEAR, J. et W.E. WeTZEL JR (1990), «Who bankroll High-Tech entrepreneurs? », Journal of Business Venturing, vol. 5, $\mathrm{n}^{\circ}$ 2, p. 77-136.

FREEAR, J., J.E. SOHL et W.E. WETZEL JR (1990), «Raising venture capital : entrepreneurs' views of the process », dans K. Vesper (éd.), Frontiers of Entrepreneurship Research, Wellesley, Mass., Babson College.

FreEAR, J. et W.E. WETZEL JR (1991), The Informal Venture Capital Market in the Year 2000, III ${ }^{e}$ Congrès annuel sur la recherche en PME, Florida State University.

FreEAR, J., J.E. SoHL et W.E. WeTzEL JR (1992), «The investment attitudes, behavior and characteristics of high net worth individuals», dans K. Vesper (éd.), Frontiers of Entrepreneurship Research, Wellesley, Mass., Babson College.

GaSton, R.S. et S. Bell (1986), Informal Risk Capital in the Sunbelt Region, Knoxille, Tenn., Applied Economics Group.

GASTON, R.S. et S. BELL (1988), «The informal supply of capital», Washington DC, Office of Economic Research, U.S. Small Business Administration.

Gaston, R.S. (1989), Finding Private Venture Capital for Your Firm: A Complete Guide, New York, J. Wiley.

GEOFFron, P. (1991), «Une analyse du processus de structuration des industries de capital de risque», Revue Internationale PME, vol. 4, n 3, p. 95-114.

HAAR, N.E., J. STARR et I.C. MACMILlAN (1988), «Informal risk capital investors: investment patterns on the East Coast of the USA», Journal of Business Venturing, vol. 3, p. 11-29.

HAKIM, C. (1989), «Identifying fast growth small firms», Employment Gazette, vol. 91, p. $39-41$.

HARRISON, R.T. et C.M. MASON (1990), «Informal risk capital in the UnitedKingdom», dans K. Vesper (éd.), Frontiers of Entrepreneurship Research, Wellesley, Mass., Babson College.

HARRISON, R.T. et C.M. MASON (1992a), «International perspectives on the supply of informal venture capital », Journal of Business Venturing, vol. 7, p. 459-475.

HARRISON, R.T. et C.M. MASON (1992b), «The role of investors in entrepreneurial companies : a comparison of informal investors and venture capitalists », dans K. Vesper (éd.), Frontiers of Entrepreneurship Research, Wellesley, Mass., Babson College. 
HARRISON, R.T. (1993), «Informal venture capital and early stages ventures: European evidence and experience», The Six Countries Program Conference, Financing the Early Stage Technology Companies in the 1990s: An International Perspective, Montréal.

JOG, V., W.M. LAWSON et A. RIDING (1991), «The venture capitalist/ entrepreneur interface: expectations, conflicts, and contracts », Journal of Small Business \& Entrepreneurship, vol. 8, no 2, p. 5-20.

KNIGHT, R.M. (1985), «The financing of small high-technology firms in Canada», Journal of Small Business \& Entrepreneurship, vol. 3, $\mathrm{n}^{0}$ 1, p. 5-17.

LANDSTRÖM, H. (1993), "Informal risk capital in Sweden and some international comparisons », Journal of Business Venturing, vol. 8, p. 525-540.

LeVRatTo, N. (1990), «Le financement des PME par les banques: contraintes des firmes et limites de la coopération », Revue Internationale PME, vol. 3, $\mathrm{n}^{\circ} 2$, p. 193-213.

Macdonald, M. ET ASSOCIÉs (1994), The Venture Capital Market in Québec, Toronto.

MASON, C.M., R.T. HARRISON et J. ChALONER (1991), Informal Risk Capital in the UK: A Study of Investors Characteristics, Investment Preferences and Investment Decision Making, Venture Finance Research Project, Document de travail $n^{0} 2$, University of Southampton and University of Ulster.

OCDE (1986), Le capital de risque: contexte, développement et actions des pouvoirs publics, Paris, Service des publications de l'OCDE.

OU, C. (1987), Holdings of Privately-Held Business Assets by American Families: Findings from the 1983 Consumer Finance Survey, Document non publié, Washington DC, Office of Economic Research, US Small Business Administration.

Postma, P.D. et M.K. Sullivan (1990), Informal Risk Capital in the Knoxville Region, Center of Excellence for New Venture Analysis, College of Business Administration, Knoxville, Tenn., The University of Tennessee.

RIDING, A.L. et D.M. SHORT (1987), «Some investors' and entrepreneurs' perceptions of the informal market for risk capital», Journal of Small Business \& Entrepreneurship, vol. 4, p. 19-30.

RIDING, A.L. et D.M. SHORT (1988), «On the estimation of the investment potential of informal investors: a capture/ recapture approach », Journal of Small Business \& Entrepreneurship, vol. 5, $\mathrm{n}^{0} 5$, p. 26-40.

Riding, A., P.D. Cin, L. Duxbury, G. Haines et R. Safrata (1993), Informal Investors in Canada: The Identification of Salient Characteristics, Rapport soumis au ministère de l'Industrie, de la Science et des Technologies, Ottawa.

RUHNKA, J.C. et J.E. YounG (1987), «A venture capital model of the development process for new ventures », Journal of Business Venturing, vol. 2, p. 167-184. 
Sullivan, M.K. et A. MiLler (1990), «Applying theory of finance to informal risk capital research: promise and problems », dans K. Vesper (éd.), Frontiers of Entrepreneurship Research, Wellesley, Mass., Babson College.

SURET, J.M. (1994), «Le Gouvernement du Québec et le financement des entreprises: les mauvaises réponses à un faux problème», dans L'État interventionniste, Vancouver, The Fraser Institute.

Suret, J.M. et L. ARNOUX (1995), L'investissement direct au Québec, Québec, Conseil de la science et de la technologie, 82 pages.

TYMES, E.R. et O.J. KRASNER (1983), «Informal risk in California», dans K. Vesper (éd.), Frontiers of Entrepreneurship Research, Wellesley, Mass., Babson College.

Venture Economics Canada Ltd (1990), Financing Early Stage Companies, Toronto, Venture Economics Canada Inc.

VERnA, G. (1994), «Légalité-légimité: la dialectique de l'informel», dans Les entreprises informelles, Collectif publié sous la direction de R. Arrelano, Y. Gasse et G. Verna, Sainte-Foy, Québec, les Presses de l'Université Laval.

WETZEL, W.E. JR (1981), «Informal risk capital in New England», dans K. Vesper (éd.), Frontiers of Entrepreneurship Research, Wellesley, Mass., Babson College.

WETZEL, W.E. JR (1983), «Angels and informal risk capital», Sloan Management Review, vol. 24, no 4, p. 21-34.

WETZEL, W.E. JR (1987), «The informal venture capital market: aspects of scale and market efficiency », Journal of Business Venturing, vol. 2, nº 4, p. 299-313.

WETZEL, W.E. JR et J. FREEAR (1994), «Promoting informal venture capital in the United States: reflections on the history of the venture capital network », dans R.T. Harrison et C.M. Mason (éd.), Informal Venture Capital: Information, Networks and Public Policy, Woodhead-Faulkner, Hemel Hempstead. 